colostrum, le $\mathrm{Cl}$ et le $\mathrm{P}$ varient dans le même sens et les rapports $\frac{\mathrm{P}}{\mathrm{Cl}}$ sont élevés. Dans certains laits, le phosphore varie en sens inverse $\mathrm{du}$ chlore et dans d'autres ses modifications ont lieu dans le même sens, mais dans des proportions différentes, si bien que même pour ces derniers laits ce rapport ne présente guère de fixité.

Le rapport $\frac{\mathrm{P}}{\mathrm{Cl}}$ peut dans certains cas servir à déceler le salage d'un lait d'origine connue, mais il présente des écarts trop considérables pour pouvoir être utilisé lorsqu'il s'agit de lait dont on ignore la provenance.

\title{
BIBLIOGRAPHIE
}

[1] Barthe et Dufinho. Dosage du chlore et du sodium dans les laits de quelques femelles de mammifères. Ann. des fals., 1928, p. 98 .

[2] Mire Sarran. Etude d'une nouvelle Constante pour déceler le salage dans lo lait. Le Lait, 1932, p. 788.

[3] Staminer. La Constante moléculaire des laits. Journal. de Pharm. de Belgique, $\mathrm{n}^{\mathrm{os}} 30$ के $35,1931$.

\section{SUR LE DOSAGE DES MATIËRES MINÉRALES DES CASÉINES INDUSTRIELLES}

\section{JEAN PIEN \\ par}

Ingénieur-chimiste (I. C.R.),

Docteur ès Sciences, Directeur et S. HERSCHDOERFER

Docteur ès Sciences

(Vienne).

des Laboratoires des « Fermiers réunis ».

Le dosage des matières minérales constitue, dans l'analyse des caséines lactiques, l'un des éléments les plus intéressants d'appréciation de la qualité du produit. On sait, en effet, qu'une caséine lactique est d'autant meilleure pour l'industrie (et surtout pour la fabrication des colles) qu'elle renferme moins de matières minérales. Il est donc intéressant de savoir exécuter correctement ce dosage.

Le but de cette étude est :

10 De démontrer que la méthode de dosage actuellement en usage est entachée de causes d'erreurs susceptibles de conduire à des interprétations fausses ;

$2^{\circ}$ De proposer une méthode exacte et rationnelle pour la détermination des matières minérales dans les caséines.

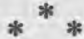




\section{$\S 1$.}

\section{LES ERREURS COMMISES DANS LE DOSAGE DES CENDRES PAR LA MÉTHODE CLASSIQUE DE CAICINATION.}

La caséine pure, théorique, ne contient pas de matières minérales. Elle renferme, en dehors de $\mathrm{C}, \mathrm{H}, \mathrm{O}, \mathrm{Az}$, du soufre et du phosphore organiques non liés à des bases minérales. La calcination d'un tel produit ne doit donc pas laisser de cendres. Pratiquement, en préparant de la caséine pure en suivant la technique de HaMMARSTEN on obtient un produit qui ne laisse que $0,1 \%$ de cendres.

La préparation de la caséine lactique industrielle est basée sur le même principe que la précipitation des caséines pures. Mais de nombreuses raisons s'opposent à ce que les produits obtenus dans l'industrie soient exempts de matières minérales :

10 Présence fréquente dans le caillé de germes protéolytiques qui communiquent au coagulum certaines des propriétés chimiques des caillés de présure ;

$2^{\circ}$ Précipitation en dehors du point isoélectrique;

$3^{\circ}$ Conditions de température et de durée optima, rarement observées ;

$4^{\circ}$ Lavages en général insuffisants.

De toutes ces circonstances. c'est peut-être la dernière qui, dans la pratique industrielle, présente la plus grande importance; car, en général, la fermentation lactique est assez lente et va assez loin pour rompre le complexe caséinate phosphocalcique, forme sous laquelle se trouve la caséine dans le lait. L'insuffisance des lavages laisse subsister dans le caillé des phosphates calciques qui appartenaient au complexe dont la caséine faisait partie dans le lait, et également des sels solubles du sérum. Il n'y a done rien d'étonnant à ce que l'on trouve, en calcinant les caséines lactiques industrielles, des chiffres parfois très supérieurs à ceux que donnent les caséines pures préparées au laboratoire.

Pour la détermination des cendres des caséines on procède d'ordinaire à une simple calcination à poids constant.

Cette manière d'opérer entraîne deux séries d'erreurs.

1. Fixation d'UNE certaine quantite de phosphore orgaNIQUE QUI NE DEVRAIT PAS FIGURER DANS LES MATIĖRES MINÉRALES.

- La calcination d'une caséine chimiquement pure ne laisse pratiquement pas de cendres. Donc, tout le phosphore organique de la molécule de caséine (dont le taux, à peu près constant, est voisin de $0,7 \%$ ) disparaît lors de la calcination. Une caséine lactique industrielle contient toujours à eôté de ce phosphore organique constitutionnel, une quantité variable de phosphore minéral extérieur à la caséine pure précipitée. Le phosphore minéral, dont la 
présence est toujours anormale et indésirable, doit être compté dans le taux de matières minérales réelles, alors que le phosphore organique ne devrait jamais être ni dosé, ni compté comme cendres. Or, il est de connaissance courante que le phosphore organique contenu dans une substance soumise à la calcination se fixe en partie sur les matières minérales ambiantes. On a d'ailleurs mis à profit cette propriété dans le dosage de certains métalloïdes (As, P, etc.) par incorporation à la matière, avant calcination, de sels minéraux en excès. Un certain nombre de techniques toxicologiques sont basées sur ce principe. L'expérience a montré qu'il fallait un gros excès de matières minérales pour la fixation totale du métalloïde pendant la calcination ; mais elle a montré également qu'en présence d'une petite quantité de bases fixes, la fixation du phosphore était loin d'être négligeable.

On est done logiquement fondé à admettre que lors de la calcination d'une caséine renfermant des matières minérales on va fixer une certaine quantité d'acide phosphorique. La pratique nous a montré que l'erreur ainsi commise pouvait atteindre de 0,3 à $0,5 \%$ et même quelquefois $1 \%$ de la caséine initiale; et la teneur en cendres annoncée est fausse par excès de cette quantité. Si la fixation du phosphore organique était totale, le problème serait grandement simplifié ; car il suffirait, pour corriger le chiffre de matières minérales, de retrancher le terme correctif à peu près constant correspondant à la teneur des caséines en $\mathrm{P}$ organique (1). (Ch. Porcher a rappelé dans son ouvrage sur "Le lait au point de vue colloïdal " de nombreux résultats de divers auteurs qui oscillent tous entre 0,7 et 0,8 de phosphore organique pour cent de caséine.) Malheureusement il n'en est rien, comme on pouvait le prévoir, en raison du taux de matières minérales insuffisant. En revanche, dans le cas des caséines présure la fixation du phosphore est à peu près complète ; en effet :

\begin{tabular}{|c|c|c|c|c|c|c|c|c|}
\hline \multirow[b]{2}{*}{ No des caséines..... } & \multicolumn{3}{|c|}{$\begin{array}{c}\text { Caséines lactiques } \\
\text { bien lavées }\end{array}$} & \multicolumn{3}{|c|}{$\begin{array}{c}\text { Caséines lactiques } \\
\text { mal lavées }\end{array}$} & \multicolumn{2}{|c|}{$\begin{array}{l}\text { Casóine } \\
\text { présure }\end{array}$} \\
\hline & 1 & 2 & 3 & 4 & 5 & 6 & 7 & 8 \\
\hline $\begin{array}{l}\mathrm{P}^{2} \mathrm{O}^{5} \text { total (attaque sul- } \\
\text { fonitrique) . . . . . . }\end{array}$ & 1,69 & 1,69 & 1,62 & 1,72 & 1,81 & 1,84 & 3,48 & 3,84 \\
\hline $\begin{array}{l}\mathrm{P}^{2} \mathrm{O}^{5} \text { dosé dans les cen- } \\
\text { dres } \ldots \ldots \ldots \ldots \ldots\end{array}$ & 0,16 & 0,17 & 0,20 & 0,22 & 0,74 & 0,43 & 3,36 & 3,92 \\
\hline
\end{tabular}

(1) Il ne faudrait pas songer à doser le phosphore dans les cendres et à le retrancher du chiffre de matières minérales car, ce faisant, on éliminerait le phosphore minéral qui doit être compté. 
Notons en passant que nous avons trouvé environ 1,70 de $\mathrm{P}^{2} \mathrm{O}^{5}$, c'est-à-dire environ 0,74 de phosphore dans les caséines les mieux lavées, teneur qui confirme les chiffres rappelés ci-dessus pour le phosphore organique des caséines pures.

Donc, le mode de calcination pure et simple utilisé actuellement pour doser les cendres des caséines, fixe une partie du phosphore organique, mais ne fixe pas tout, loin s'en faut, sauf dans le cas des caséines présure.

\section{FiXATION, DANS LES CENDRES, DE MATIERES ORGANIQUES} INCOMPLÈTEMENT BRULÉEs. - La deuxième cause d'erreur réside dans le fait que la calcination d'une caséine lactique est une opération très pénible. Il est fort difficile de détruire tout le charbon ; les cendres restent toujours grises et bien souvent noires. Si on élève la température, on fond certains sels alcalins et la substance enrobée échappe à la combustion. De nombreuses reprises à l'eau chaude avec calcination séparée du résidu insoluble et de l'extrait sec des eaux de lavage permet d'obtenir des cendres non faussées. Mais e'est là une opération fastidieuse et longue. Il est plus facile, pour brûler toute la matière organique, de reprendre les cendres par de l'acide nitrique fumant, évaporer au bain de sable et calciner à nouveau. On arrive ainsi après plusieurs traitements à obtenir des cendres blanches et l'erreur commise par les modifications chimiques apportées aux matières minérales au cours de ces traitements est nulle, ear ces modifications elles-mêmes sont nulles.

Quoi qu'il en soit, la perte de poids due à la disparition des matières organiques est parfois très notable. En effet :

\begin{tabular}{|c|c|c|c|c|c|c|}
\hline \multirow[b]{2}{*}{ No des caséines lactiques. } & \multicolumn{3}{|c|}{ Caséines bien lavées } & \multicolumn{3}{|c|}{ Caséines mal lavées } \\
\hline & 1 & 2 & 3 & 4 & 5 & 6 \\
\hline Cendres classiques .... & 0,42 & 0,52 & 0,48 & 1,32 & 2,62 & 1,42 \\
\hline Cendres nitriques ..... & 0,24 & 0,26 & 0,36 & 0,94 & 2,10 & 1,20 \\
\hline $\begin{array}{l}\text { Perte totale (ou sur- } \\
\text { charge des cendres } \\
\text { classiques en matières } \\
\text { organiques non brû- }\end{array}$ & 0.18 & 0.26 & 0.12 & 0.38 & 0.52 & 0.22 \\
\hline
\end{tabular}

Ces surcharges de carbone, plus ou moins visibles à cause des enrobements de sels fondus, sont donc très importantes.

En résumé : le dosage des cendres des caséines lactiques par la 
méthode classique de calcination simple est entachée de deux causes d'erreur :

$1^{\circ}$ Fixation d'acide phosphorique organique ;

2. Conservation d'une quantité appréciable de matières organiques incomplètement brûlées.

Ces deux causes agissent dans le même sens et les cendres ainsi déterminées pèchent par excès.

$$
\begin{aligned}
& *^{*} * \\
& \S 2 .
\end{aligned}
$$

REMÈDE A CETTE SITUATION. - DOSAGE CORRECT DES MATIĖRES MINÉRALES DES CASÉINES LACTIQUES.

1. PRINCIPE. - Comment éliminer ces deux causes d'erreur ?

L'usage de l'acide nitrique fort sur les cendres après calcination pourrait, sans doute, éliminer les matières non brûlées. Mais cette opération n'apporte aucun remède à la fixation du phosphore organique. Les "cendres nitriques " ne résolvent point la question. Il n'y a en réalité qu'un moyen à employer : c'est d'incorporer à la caséine avant calcination un excès connu de matières minérales fixes bien choisies. De cette façon on pourra fixer la totalité du phosphore organique et d'autre part l'expérience montre que tout le charbon brûlera aisément. (On sait déjà que dans le cas de caséines-présure, les cendres sont toujours très blanches, à cause précisément de la présence de matières minérales en excès.)

Nous aurons ainsi :

10 Supprimé complètement la cause d'erreur due aux matières organiques non brûlées ;

20 Remplacé la fixation partielle, variable et toujours inconnue du phosphore organique par une fixation totale de cet élément, dont le pourcentage, qui varie très peu, est connu.

Il suffira enfin de retrancher de ces cendres totales :

a) D'abord les matières minérales en excès introduites avant calcination ;

b) Ensuite le terme fixe 1,60 (1) représentant le taux de phosphore organique, commun à toutes les caséines pures, exprimé ici nécessairement en anhydride phosphorique $\mathrm{P}^{2} \mathrm{O}^{5}$, forme sous laquelle il s'est fixé aux bases au cours de la calcination. L'incertitude qui régnera sur le chiffre ainsi obtenu sera de l'ordre de $0,1 \%$. Mais le résultat sera beaucoup plus près de la vérité que celui que donne la calcination pure et simple.

(1) Nous proposons d'adopter le terme 1,60 qui correspond ò 0,70 de phosphore, chiffre moyen des auteurs. 
Le principe de cette méthode est dû à SHAw (Journ. Ind. Eng. Chem., vol. 12, 1920, p. 1168).

2. RÉalisation. - La base fixe choisie est la chaux, introduite dans la caséine avant calcination sous la forme d'une solution d'acétate. Cette solution est titrée dans les conditions du dosage, par calcination d'un volume connu de la solution du sel. L'acétate peut être remplacé avantageusement par le nitrate, dont la solution se conserve mieux, et dont l'emploi facilite mieux la combustion de la caséine.

\section{On opère ainsi :}

5 gr. de caséine finement broyée (1) sont mélangés (dans une capsule de quartz ou de platine) à $5 \mathrm{~cm}^{3}$ de solution d'acétate de calcium à $30 \mathrm{gr}$. de $\mathrm{CaO}$ par litre environ, exactement titrée par calcination. On laisse gonfler la caséine pendant $1 / 2$ heure, pour qu'elle s'imprègne parfaitement du sel de chaux. On sèche à l'étuve et on calcine, modérément d'abord, pour finir à température plus élevée (on n'a plus à redouter la fusion des cendres).

Connaissant le titre en CaO de la solution calcique, on corrige les cendres de la quantité de base introduite.

Enfin, le chiffre obtenu est diminué du terme fixe 1,60 correspondant au $\mathrm{P}^{2} \mathrm{O}^{5}$ d'origine organique fixé pendant la calcination.

3. RÉSULTATS OBTENUS. - 10 Il s'agissait d'abord de vérifier que tout le phosphore organique est bien fixé au cours de cette calcination en présence d'un excès de base.

Il en est bien ainsi. En effet :

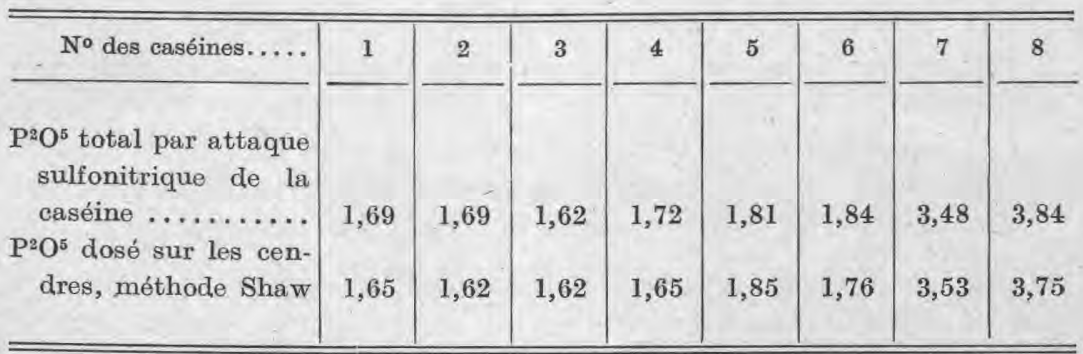

Done les "cendres Shaw ", c'est-à-dire le chiffre obtenu par la méthode décrite ci-dessus, et corrigé seulement des matières minérales introduites, donnent bien les cendres totales de la caséine y compris la totalité du phosphore organique.

$2^{\circ}$ La soustraction du terme constant $1,60\left(\mathrm{P}^{2} \mathrm{O}^{5}\right.$ organique de la caséine) doit, à priori, donner un résultat exact.

(1) Si on a affaire à une caséine non fine (exemple : refus du tamis 60), on peutnéanmoins se dispenser de la broyer, en ajoutant $5 \mathrm{~cm}^{3}$ d'eau en plus des $5 \mathrm{~cm}^{3}$ de solution d'acétate de calcium, pour faciliter l'imprégnation. 
Ce résultat est le suivant pour les caséines examinées :

\begin{tabular}{|c|c|c|c|c|c|c|c|c|}
\hline No des caséines...... & 1 & 2 & 3 & 4 & 5 & 6 & 7 & 8 \\
\hline $\begin{array}{c}\text { Cendres elassiques par } \\
\text { ealcinationsimple }\end{array}$ & 0,42 & 0,52 & 0,48 & 1,32 & 2,62 & 1,42 & 1,40 & 8,29 \\
\hline Cendres Shaw $\quad . . .$. & 1,86 & 2,02 & 2,12 & 2,12 & 2,66 & 2,30 & 7,76 & 8,54 \\
\hline $\begin{array}{l}\text { Cendres Shaw corri- } \\
\text { gées du } \mathrm{P}^{2} \mathrm{O}^{6} \text { organi- } \\
\text { que (cendres réelles) }\end{array}$ & 0,26 & 0,42 & 0,52 & 0,52 & 1,06 & 0,70 & 6,16 & 6,94 \\
\hline
\end{tabular}

Bien qu'à priori ces chiffres doivent correspondre à la teneur exacte des caséines en matières minérales vraies, nous avons un moyen pratique de vérifier leur exactitude :

Les cendres Shaw titrent tout le phosphore organique. Nous connaissons d'autre part le phosphore total, que nous avons dosé soit par attaque directe de la caséine, soit par dosage sur le résidu de calcination des cendres Shaw (cendres reprises par HCl et dosage par le phosphate ammoniaco-magnésien en présence d'un excès de citrate d'ammoniaque selon le procédé classique). En retranchant cet acide phosphorique total des cendres Shaw nous commettrions une erreur, ear une partie du phosphore est d'origine minérale et doit figurer au résultat. Mais la majeure partie du phosphore minéral des caséines lactiques est d'origine calcique. Dosons done la chaux sur les cendres classiques obtenues par calcination simple et calculons la quantité d'acide phosphorique correspondant à cette chaux dans la forme tricalcique. Si nous retranchons cette quantité d'acide phosphorique minéral "calculé ", de l'acide phosphorique total dosé, nous obtiendrons l'acide phosphorique organique initial à peu près exactement. En retranchant ce chiffre des cendres Shaw, nous devons retrouver à nouveau les cendres réelles (1).

\begin{tabular}{|c|c|c|c|c|c|c|}
\hline \multirow[b]{2}{*}{$N^{o}$ des caséines..... } & \multicolumn{3}{|c|}{ Cassénes lactiques bien lavées } & \multicolumn{3}{|c|}{ Caséines lactiques mal lavées } \\
\hline & 1 & 2 & 3 & 4 & 5 & 6 \\
\hline $\begin{array}{r}\mathrm{P}^{2} \mathrm{O}^{5} \text { total dosé sur les } \\
\text { cendres Shaw (A) ... }\end{array}$ & 1,65 & 1,62 & 1,62 & 1,65 & 1,85 & 1,76 \\
\hline $\begin{array}{l}\mathrm{CaO} \text { totale sur les cen- } \\
\text { dres classiques...... }\end{array}$ & traces & traces & traces & 0,20 & 0,30 & 0,14 \\
\hline $\mathrm{P}^{2} \mathrm{O}^{5}$ correspondant (B) & - & - & - & 0,17 & 0,25 & 0,12 \\
\hline $\mathrm{P}^{2} \mathrm{O}^{5}$ organique $(\mathrm{A}-\mathrm{B})(\mathrm{C})$ & 1,65 & 1,62 & 1,62 & 1,48 & 1,60 & 1,64 \\
\hline Cendres Shaw (D) .... & 1,86 & 2,02 & 2,12 & 2,12 & 2,66 & 2,30 \\
\hline Cendres réelles (D-C) .. & 0,21 & 0,40 & 0,50 & 0,64 & 1,06 & 0,66 \\
\hline
\end{tabular}

(1) Ce raísonnement n'est pas exact pour les caséines-présure où toute la chaux n'est pas liée à de l'acide phosphorique. 
Ce tableau nous montre que le terme 1,60 , qui a été choisi comme teneur moyenne des caséines lactiques en $\mathrm{P}^{2} \mathrm{O}^{5}$ organique, peut être considéré comme exact. Dans ces conditions, on doit avoir une assez bonne concordance entre les cendres réelles calculées à l'aide du terme constant 1,60 et les cendres réelles dosées en passant par les déterminations de l'acide phosphorique et de la chaux.

C'est en effet ce qui ressort du tableau suivant, où l'écart avec les cendres "méthode classique » est, en outre, mis nettement en évidence :

\begin{tabular}{|c|c|c|c|c|c|c|}
\hline No des casóines..... & 1 & 2 & 3 & 4 & 5 & 6 \\
\hline $\begin{array}{c}\text { Cendres réelles calcu- } \\
\text { lées (1) } \ldots \ldots \ldots \ldots \ldots\end{array}$ & 0,26 & 0,42 & 0,52 & 0,52 & 1,06 & 0,70 \\
\hline Cendres réelles dosées (2) & 0,21 & 0,40 & 0,50 & 0,64 & 1,06 & 0,66 \\
\hline Cendres classiques .... & 0,42 & 0,52 & 0,48 & 1,32 & 2,62 & 1,42 \\
\hline $\begin{array}{l}\text { Erreur en trop des cen- } \\
\text { dres classiques...... }\end{array}$ & 0,21 & 0,12 & - & 0,68 & 1,56 & 0,76 \\
\hline
\end{tabular}

La surcharge des cendres classiques est très irrégulière; ce qui montre que la calcination pure et simple n'offre aucune garantie et peut mener aux conclusions les plus fantaisistes.

4. commentaires. - $1^{0}$ Le tableau précédent exprime nettement que bien souvent les cendres des caséines lactiques déterminées par la méthode classique de calcination, sont entachées d'erreur par excès.

$2^{\circ} \mathrm{Il}$ serait fastidieux dans la pratique de doser l'acide phosphorique total, puis la chaux totale, pour évaluer le terme correctif correspondant à l'acide phosphorique organique fixé pendant la calcination selon SHAw.

$3^{\circ}$ Il est beaucoup plus simple et pratiquement aussi exact d'adopter le terme correctif de 1,60, correspondant à l'acide phosphorique organique de la caséine, que la calcination selon SHAw aura entièrement fixé.

A titre documentaire, voici un certain nombre de résultats obtenus sur différentes caséines lactiques, d'une part par la méthode

(1) Voir premier tableau, page 1087.

(2) Voir tableau précédent. 
classique de calcination pure et simple, d'autre part par la méthodeShaw modifiée :

\begin{tabular}{|c|c|c|c|c|c|c|}
\hline & No des caséines & $\begin{array}{c}\text { Cendres } \\
\text { classi- } \\
\text { ques }\end{array}$ & $\begin{array}{c}\text { Méthode } \\
\text { Shaw } \\
\text { modifiée }\end{array}$ & No des caséines & $\begin{array}{c}\text { Cendres } \\
\text { classi- } \\
\text { ques }\end{array}$ & $\begin{array}{l}\text { Méthode } \\
\text { Shaw } \\
\text { modifiée }\end{array}$ \\
\hline 1 & $\ldots \ldots \ldots \ldots \ldots$ & 2,04 & 0,56 & $21 \ldots \ldots \ldots \ldots \ldots$ & 2,16 & 0,58 \\
\hline 2 & $\ldots \ldots \ldots \ldots \ldots$ & 2,00 & 0,58 & $22 \ldots \ldots \ldots \ldots \ldots$ & 2,10 & 0,78 \\
\hline 3 & $\ldots \ldots \ldots \ldots \ldots$ & 1,90 & 0,68 & $23 \ldots \ldots \ldots \ldots \ldots$ & 2,14 & 0,68 \\
\hline 4 & $\ldots \ldots \ldots \ldots \ldots$ & 1,90 & 0,56 & $24 \ldots \ldots \ldots \ldots$ & 2,68 & 1,22 \\
\hline 5 & $\ldots \ldots \ldots \ldots \ldots$ & 2,12 & 0,60 & $25 \ldots \ldots \ldots \ldots$ & 2,20 & 0,78 \\
\hline 6 & $\ldots \ldots \ldots \ldots \ldots$ & 1,94 & 0,50 & $26 \ldots \ldots \ldots \ldots$ & 2,06 & 0,56 . \\
\hline 7 & $\ldots \ldots \ldots \ldots \ldots$ & 1,74 & 0,48 & $27 \ldots \ldots \ldots \ldots$ & 1,82 & 0,68 \\
\hline 8 & $\ldots \ldots \ldots \ldots \ldots$ & 2,06 & 0,48 & $28 \ldots \ldots \ldots \ldots$ & 2,30 & 0,88 . \\
\hline 9 & $\ldots \ldots \ldots \ldots \ldots$ & 2,30 & 0,60 & $29 \ldots \ldots \ldots \ldots$ & 2,56 & 1,08 . \\
\hline 10 & $\ldots \ldots \ldots \ldots \ldots$ & 1,20 & 0,18 & $30 \ldots \ldots \ldots \ldots$ & 2,58 & 1,00 \\
\hline 11 & $\ldots \ldots \ldots \ldots \ldots$ & 2,02 & 0,48 & $31 \ldots \ldots \ldots \ldots$ & 1,86 & 1,00 \\
\hline 12 & $\ldots \ldots \ldots \ldots \ldots$ & 2,26 & 0,62 & $32 \ldots \ldots \ldots \ldots \ldots$ & 1,86 & 0,58 \\
\hline 13 & $\ldots \ldots \ldots \ldots \ldots$ & 2,00 & 0,46 & $33 \ldots \ldots \ldots \ldots \ldots$ & 1,82 & 0,48 . \\
\hline 14 & $\ldots \ldots \ldots \ldots \ldots$ & 2,12 & 0,68 & $34 \ldots \ldots \ldots \ldots$ & 1,98 & 0,64 \\
\hline 15 & $\ldots \ldots \ldots \ldots \ldots$ & 1,90 & 0,40 & $35 \ldots \ldots \ldots \ldots \ldots$ & 1,50 & 0,38 \\
\hline 16 & $\ldots \ldots \ldots \ldots \ldots$ & 1,62 & 0,54 & $36 \ldots \ldots \ldots \ldots \ldots$ & 1,60 & 0,38 : \\
\hline 17 & $\ldots \ldots \ldots \ldots \ldots$ & 1,72 & 0,52 & $37 \ldots \ldots \ldots \ldots$ & 1,88 & 0,48 \\
\hline 18 & $\ldots \ldots \ldots \ldots \ldots$ & 1,78 & 0,48 & $38 \ldots \ldots \ldots \ldots \ldots$ & 2,16 & 0,60 \\
\hline 19 & $\ldots \ldots \ldots \ldots \ldots$ & 2,18 & 0,66 & $39 \ldots \ldots \ldots \ldots$ & 1,80 & 0,76 \\
\hline 20 & $\ldots \ldots \ldots \ldots \ldots$ & 1,64 & 0,16 & $40 \ldots \ldots \ldots \ldots$ & 2,18 & 0,82 \\
\hline
\end{tabular}

$\S 3$.

CONCLUSIONS PRATIQUES.

$1^{0}$ Nous proposons de remplacer la calcination habituelle, parla calcination en présence d'un excès de base minérale (méthode Shaw), qui permet de brûler intégralement toutes les matières organiques, et en outre de fixer la totalité du phosphore organique de la caséine.

$2^{\circ}$ Nous proposons de faire subir aux résultats obtenus selon SHAw, la correction soustractive de $1,60 \%$, qui correspond pratiquement à la teneur en acide phosphorique organique, à peu près constante, de la caséine du lait de vache - cet acide phosphorique de la. molécule protéique ne devant pas figurer dans le chiffre des matières: minérales de la caséine. 